Original

\title{
Lack of Initiating Activity of Kojic Acid on Hepatocarcinogenesis in F344 Rats
}

\author{
Takao Watanabe ${ }^{1}$, Taeko Mori ${ }^{1}$, Yasuki Kitamura ${ }^{2}$, Takashi Umemura ${ }^{2}$, \\ Miwa Okamura $^{1}$, Yoko Kashida ${ }^{1}$, Akiyoshi Nishikawa ${ }^{2}$, \\ Masao Hirose ${ }^{2}$, and Kunitoshi Mitsumori ${ }^{1}$ \\ ${ }^{1}$ Laboratory of Veterinary Pathology, Tokyo University of Agriculture and Technology, 3-5-8 Saiwai-cho, Fuchu, Tokyo \\ 183-8509, Japan \\ ${ }^{2}$ Division of Pathology, National Institute of Health Sciences, 1-18-1 Kamiyoga, Setagaya-ku, Tokyo 158-8501, Japan
}

\begin{abstract}
Kojic acid (KA) has been used as a food additive for preventing enzymatic browning of crustaceans and as a cosmetic agent for skin whitening. To date, it has been reported that female $\mathrm{B} 6 \mathrm{C} 3 \mathrm{~F} 1$ mice receiving $3 \% \mathrm{KA}$ in the diet were found to develop hepatocellular tumors, but the underlying mechanism of liver tumorigenicity is still not clear. In the present experiments, in order to investigate possible liver initiation activity, partially hepatectomized male F344 rats received a single oral dose of 0,1000 and $2000 \mathrm{mg} / \mathrm{kg}$ body weight of KA followed by dietary administration of $0.015 \%$ of $\mathrm{N}$-2-acetylaminofluorene (2-AAF) for 2 weeks and a single $0.8 \mathrm{~mL} / \mathrm{kg}$ body weight dose of $\mathrm{CCl}_{4}$. Furthermore, male F344 rats were fed a diet containing 0 or 2\% KA for 3, 7 and 28 days, and the 8-oxodeoxyguanosine (8-OxodG) levels in nuclear DNA were measured to examine the formation of oxidative DNA adduct and cell proliferating activities of hepatocytes in the liver. In the liver initiation assay, there were no significant differences in the number or area of glutathione S-transferase placental form (GST-P) positive foci, putative preneoplastic lesions, between the KA-treated and control groups. Cell proliferation of hepatocytes in rats given KA for 3 and 7 days was significantly increased as compared with the relevant control values, but no significant elevation in 8-OxodG levels was apparent. The results of the present study suggest that KA has neither liver initiation activity nor capability of 8-OxodG formation, but some evidences suggestive of liver tumor promoting effects in rats.
\end{abstract}

(J Toxicol Pathol 2005; 18: 79-84)

Key words: Kojic acid, hepatocarcinogenicity, liver initiation assay, F344 rat

\section{Introduction}

Kojic acid (KA; 5-hydroxy-2-(hydroxymethyl)-4pyrone) is a secondary metabolic product of various species of Aspergillus and Penicillium ${ }^{1,2}$. It is known that this acid inhibits polyphenoloxidase (tyrosinase) in mushrooms ${ }^{3}$, potatoes, apples and crustaceans ${ }^{4}$. Since polyphenol oxidase catalyzes the conversion of tyrosine to melanin via 3,4dihydroxyphenylalanine and dopaquinone $\mathrm{e}^{3,5}$, KA has been used as an inhibitor of polyphenol oxidase in foods and as a food additive for preventing enzymatic browning of raw crabs and shrimps. In addition, because of its excellent skin whitening properties ${ }^{6,7}$ and inhibitory actions on human melanocyte tyrosinase ${ }^{8}$, KA has been distributed as a

Received: 10 December 2004, Accepted: 10 March 2005 Mailing address: Kunitoshi Mitsumori, Laboratory of Veterinary Pathology, Faculty of Agriculture, Tokyo University of Agriculture and Technology, 3-5-8 Saiwai-cho, Fuchu-city, Tokyo 183-8509, Japan

TEL \& FAX: 81-42-367-5771

E-mail: mitsumor@cc.tuat.ac.jp cosmetic agent for the purpose of skin lightening.

It has been reported that hepatocellular tumors were induced in $\mathrm{B} 6 \mathrm{C} 3 \mathrm{~F} 1$ mice fed diet containing 3\% KA for 20 months 9 . In our previous study in which diet containing 0 , 1.5 or $3 \% \mathrm{KA}$ was administered to heterozygous $p 53$ deficient mice of the CBA strain [p53 (+/-) mice] and their wild-type littermates $[p 53(+/+)$ mice] for 26 weeks, the incidences of hepatocellular adenomas as well as altered hepatocellular foci were increased in $p 53(+/-)$ and $p 53(+/+)$ mice, with and without initiation, as compared with untreated control mice ${ }^{10}$. In addition, the incidences of focal hepatocellular necrosis and inflammatory cell infiltration in the liver appeared to be increased by the 1.5 and $3 \% \mathrm{KA}$ diets. Since no spontaneous hepatocellular foci were observed in control animals in our earlier 26-week studies using $p 53(+/-)$ and $p 53(+/+)$ mice $^{11-13}$, it was considered that these proliferative lesions in the liver were attributable to KA. In addition, in another study in which F344 rats received diet containing 2 or $0 \%$ KA for 20 weeks with or without initiation treatment of $N$-bis(2hydroxypropyl)nitrosamine (DHPN), the numbers of 
glutathione S-transferase placental form (GST-P) positive foci were significantly increased in the KA groups as compared with the corresponding control group ${ }^{14}$. This finding suggested the possibility that KA also has carcinogenic potential in the liver of rats.

Regarding genotoxicity, a number of previous in vitro genotoxicity tests of KA showed positive results in the reverse gene mutation assay (Ames test) using microorganisms in the presence or absence of rat S9 mix fraction $^{15,16}$, and in DNA-damaging and clastogenic ability in cultured rat liver cells ${ }^{17,18}$, as well as sister chromatid exchange and chromosomal aberration in Chinese hamster ovary cells ${ }^{16}$. However, it remains uncertain whether or not the induction of hepatocellular proliferative lesions in rats as well as $p 53(+/-)$ and $p 53(+/+)$ mice was the result of genotoxicity of KA.

For further assessment, a liver initiation assay, sensitive for the detection of genotoxic carcinogens in F344 rats ${ }^{19,20}$, and a 28-day feeding study were performed to evaluate the liver initiating potential of the compound and to examine the formation of oxidative DNA adduct and cell proliferating activity in the livers of F344 rats exposed to KA.

\section{Materials and Methods}

\subsection{Test substance}

Kojic acid (KA; more than $99.5 \%$ pure), kindly provided by Alps Pharmaceutical Industry Co., Ltd. (Gifu, Japan), was admixed with powdered basal diet (CRF-1, Oriental Yeast Co., Ltd., Tokyo, Japan) to give an average concentration of $2 \%$. Diets were prepared at least bi-weekly and stored until use at $5^{\circ} \mathrm{C}$.

\section{Experimental animals}

Sixty male F344 rats, thirty of five weeks of age (28day study) and thirty of seven weeks of age (5-week study), were purchased from Japan SLC, Inc., Shizuoka, Japan, and used after an acclimatization period of one week. Rats were housed at a maximum of 5 per plastic cage with wood chips for bedding and at a maximum of 3 per steel cage, respectively, under standard conditions (room temperature, $23 \pm 2{ }^{\circ} \mathrm{C}$; relative humidity, $55 \pm 5 \%$; 12 h-light: 12 h-dark cycle), and given a pulverized basal diet (Oriental CRF-1, Oriental Yeast Co., Ltd.) and tap water ad libitum. The bedding was changed twice a week. The experiment was carried out in accordance with the Guide for Animal Experimentation of Tokyo University of Agriculture and Technology.

\section{Twenty eight-day dietary administration study in F344 rats}

Thirty male rats were split into two groups and fed a diet containing 0 or $2 \% \mathrm{KA}$ for 3, 7 and 28 days. Five rats were sacrificed in each group under ether anesthesia at each end point. All animals were injected with bromodeoxyuridine (BrdU: $100 \mathrm{mg} / \mathrm{kg}$, Sigma Chemical Co., St Louis, MO) i.p. once a day for the final 2 days of the exposure and once on the day of termination, 2 hours before sacrifice. Body weight was measured on the day of necropsy in order to calculate the liver / body weight ratio. At autopsy, the livers were immediately removed and weighed, and slices taken from each lobe were fixed in $10 \%$ buffered formalin for BrdU immunostaining. The remaining pieces of the livers were frozen in liquid nitrogen and stored at $80^{\circ} \mathrm{C}$ until measurement of 8-oxodeoxyguanosine (8OxodG) in nuclear DNA. BrdU immunostaining was performed using the avidin-biotin complex method, and for each animal at least 3000 hepatocytes were counted. Labeling indices (LIs) were calculated as the percentages of cells positive for BrdU incorporation divided by the total number of cells counted. Nuclear DNA was extracted with a DNA Extracter WB kit (Wako Pure Chemical Industries, Ltd., Osaka, Japan), and digested to deoxynucleotides with nuclease P1 (Yamasa Shoyu Co., Ltd., Chiba, Japan) and alkaline phosphatase (Sigma Chemical Co., St Louis, MO). 8-OxodG levels (8-OxodG/105 deoxyguanosine) were assessed by high-performance liquid chromatography (HPLC) with an electrochemical detection system (ECD) (HPLC pump 420, Kontron AG, Zurich Inst., Switzerland; ECD Coulochem Model-5100A, ESA, Bedford, MA).

\section{Five-week, 2-stage liver initiation assay in male F344} rats

The in vivo short-term liver initiation assay was performed according to the method of Tsuda et al. ${ }^{19}$. All animals were subjected to two-thirds partial hepatectomy on day 0, divided into 3 groups (Group 1, 8; Group 2, 12; Group $3,10)$ and $12 \mathrm{hr}$ after the completion of surgery, were treated once orally with vehicle $(0.5 \%$ carboxymethyl cellulose, $\mathrm{CMC}$ ), 1000 (half of the $\mathrm{LD}_{50}$ value) and $2000 \mathrm{mg} / \mathrm{kg}\left(\mathrm{LD}_{50}\right.$ value of KA), respectively. The vehicle and suspensions of KA were given orally at a volume of $10 \mathrm{~mL} / \mathrm{kg}$ body weight. Subsequently, the rats were fed basal diet for 2 weeks, and then diet containing $0.015 \%$ of $N$-2-acetylaminofluorene (2AAF, Japan Clea Co., Ltd., Osaka, Japan) for the following 2 weeks. Three weeks after KA administration, all animals received a single $0.8 \mathrm{~mL} / \mathrm{kg}$ body weight dose of $\mathrm{CCl}_{4}$. At the end of week 5 , the survivors were sacrificed under ether anesthesia, and slices of all liver lobes were fixed in $10 \%$ neutral buffered formalin, embedded in paraffin, sectioned, and stained immunohistochemically for glutathione Stransferase placental form (GST-P), a marker of preneoplastic foci in the rat liver, using the avidin-biotin complex method. Additionally, hematoxylin-eosin staining of serial sections was performed for microscopic examination. With a computer-assisted image analyzer (NIH image), we measured the area and number of GST-P positive foci ( $>0.1 \mathrm{~mm}$ in diameter) and the total area of each liver section examined in each animal, and then calculated the mean area and number of GST-P positive foci per unit area of all the liver sections examined.

\section{Statistical analysis}

The data obtained from both experiments were 
expressed as mean \pm S.D. and analyzed by one-way analysis of variance for homogeneity followed by Dunnett's test ${ }^{21}$ for comparison with the $0 \% \mathrm{KA}$ control group. For the initiation assay, the significance of differences between the control and KA groups was determined by Student's $t$-test ${ }^{22}$. A $p$ value less than 0.05 was considered statistically significant in both cases.

\section{Results}

\section{Twenty eight-day dietary administration study in F344} rats

Body weight gain of the $2 \%$ KA group was significantly decreased on Day 28 as compared to the control group (Fig. 1). Relative liver weights of the $2 \%$ KA groups were significantly increased as compared to those of the control group at each necropsy point (Fig. 2). The LIs of hepatocytes of rats exposed to KA in the diet for $3(3.44 \pm$ $1.13 \%)$ and $7(1.32 \pm 0.68 \%)$ days were significantly increased as compared with the relevant control values (1.88 $\pm 0.64,0.18 \pm 0.10 \%$ ), but there were no changes in the LIs between the groups (KA: $0.31 \pm 0.31$, Control: $0.28 \pm$ $0.19 \%$ ) on Day 28 (Fig. 3). 8-OxodG levels in the liver DNA of rats given KA were slightly higher than the relevant control values throughout the experimental period (Day 3: KA $0.58 \pm 0.15$, Control 0.52 \pm 0.18 ; Day 7: KA $0.73 \pm 0.26$, Control 0.58 \pm 0.18 ; Day 28: KA $0.65 \pm 0.12$, Control $0.53 \pm$ 0.068 -OxodG $/ 10^{5} \mathrm{dG}$ ), but without statistical significance (Fig. 4).

\section{Liver initiation assay in $F 344$ rats}

One rat in the control group died during the experimental period. On immunohistochemical examination, no significant differences were observed in the mean area (Control $1.53 \pm 0.74 ; 1000 \mathrm{mg} / \mathrm{kg} \mathrm{KA} 1.69 \pm 0.97$; $2000 \mathrm{mg} / \mathrm{kg} \mathrm{KA} 1.91 \pm 1.72$ ) or number (Control $4.38 \pm$

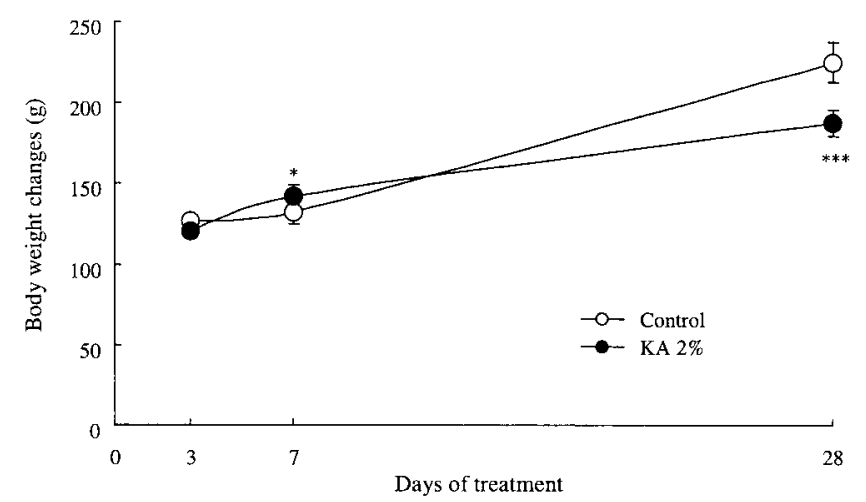

Fig. 1. Mean body weight changes in F344 rats treated with $2 \%$ Kojic acid. Five rats each were measured on Day 3,7 and 28. *: $\mathrm{p}<$ $0.05, * * *: \mathrm{p}<0.001$, Significantly different from the corresponding control group.

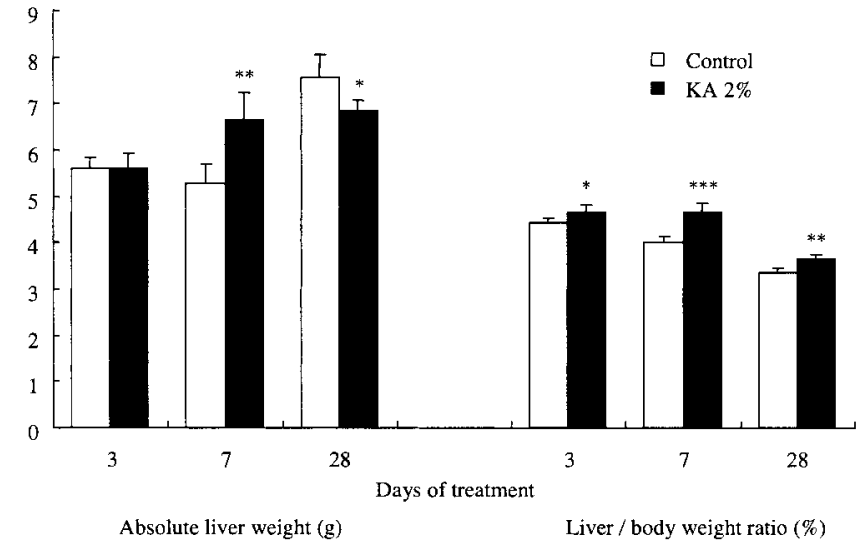

Fig. 2. Absolute liver weights and liver / body weight ratio of F344 rats treated with $2 \% \mathrm{KA}$ for 3,7 and 28 days. $*$ : $\mathrm{p}<0.05, * *$ : $\mathrm{p}<0.01, * * *: \mathrm{p}<0.001$, Significantly different from the corresponding control group.

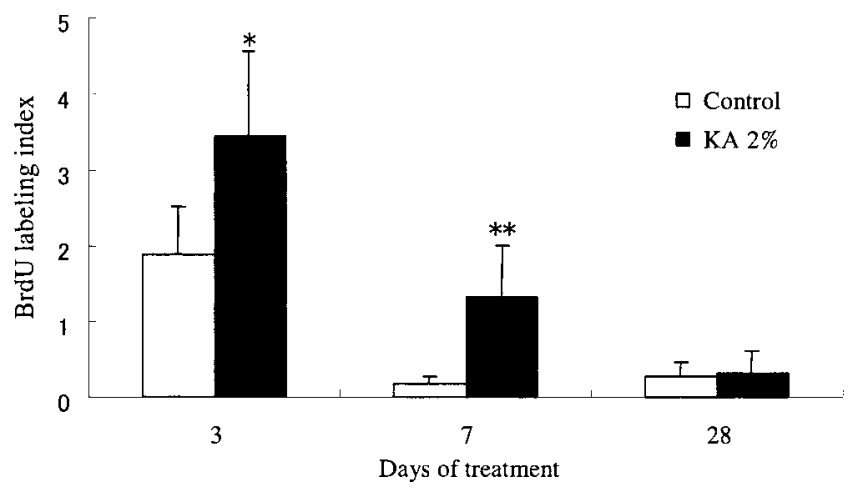

Fig. 3. BrdU labeling indices of hepatocytes of F344 rats treated with vehicle (open column) or $2 \%$ Kojic acid (closed column) for 3,7 and 28 days. *: $\mathrm{p}<0.05, * *: \mathrm{p}<0.01$, Significantly different from the corresponding control group.

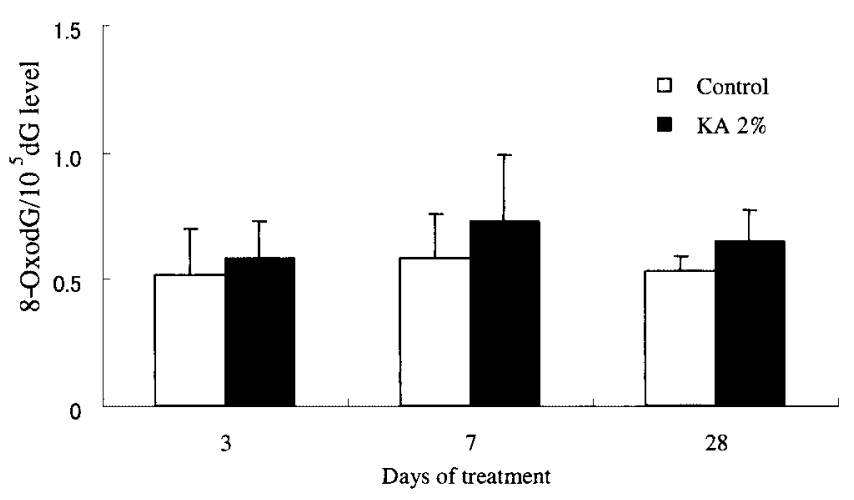

Fig. 4. 8-OxodG levels in liver DNA of F344 rats treated with vehicle (open column) or $2 \%$ Kojic acid (closed column) for 3,7 and 28 days. 

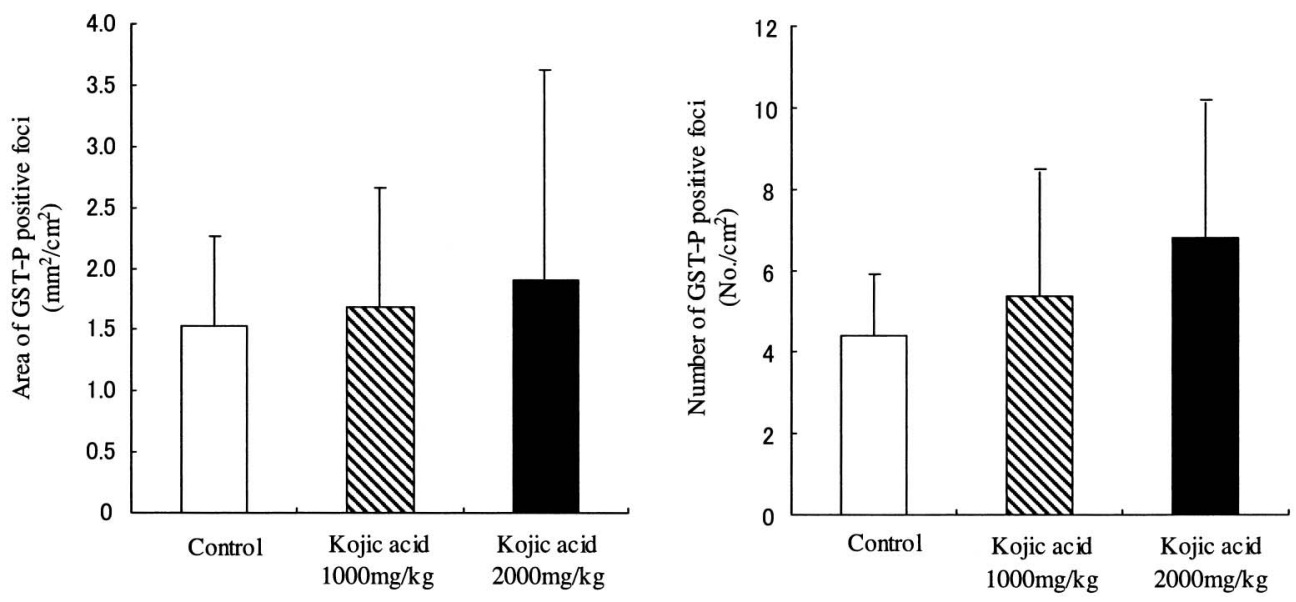

Fig. 5. Area and number of GST-P positive liver cell foci $(>0.1 \mathrm{~mm}$ in diameter) in partially hepatectomized rats treated with vehicle (open column) or kojic acid (striped or closed columns). Data represent the mean \pm S.D. of 7,12 and 10 animals.

$1.51 ; 1000 \mathrm{mg} / \mathrm{kg} \mathrm{KA} 5.36 \pm 3.04 ; 2000 \mathrm{mg} / \mathrm{kg} \mathrm{KA} 6.80 \pm$ 3.32) of GST-P positive foci between the KA and control groups (Fig. 5).

\section{Discussion}

Fujimoto et al. ${ }^{9}$ first reported that hepatocellular tumors were induced in female $\mathrm{B} 6 \mathrm{C} 3 \mathrm{~F} 1$ mice receiving $3 \% \mathrm{KA}$ in the diet for 20 months, but they did not pay special attention to the positive result of the liver tumorigenicity of KA. In our previous study in which $p 53(+/-)$ mice, that have high susceptibility to genotoxic carcinogens ${ }^{11,23-25}$, and $p 53(+/+)$ mice were fed diet containing $0,1.5$ or $3 \% \mathrm{KA}$ for 26 weeks, the incidence of hepatocellular adenomas and altered hepatocellular foci were increased in the 1.5 and $3 \% \mathrm{KA}$ groups of $p 53(+/-)$ mice $(7 / 10$ and $5 / 10)$ and $p 53(+/+)$ CBA mice $(2 / 10$ and $5 / 10)$ as compared to the control group ( $0 /$ $10)^{10}$. In addition, in the rat two-stage carcinogenesis model using DHPN, or the medium-term liver bioassay initiated with DEN, KA demonstrated clear promoting activity of hepatocarcinogenesis ${ }^{14}$. Furthermore, although the numbers and areas were small, KA increased GST-P-positive foci after 20 -week dietary administration of 2\% KA without any prior initiation treatment ${ }^{14}$. These results indicate that KA is a promoter and possibly also a complete hepatocarcinogen in rats as well as in mice. With respect to the genotoxicity, KA has been shown not to be mutagenic to Salmonella typhimurium TA1537 or TA1538 strains, but it was mutagenic to the TA100, TA1535 and TA98 strains in the presence or absence of rat $\mathrm{S} 9 \mathrm{mix}^{15,16}$. While positive and negative responses were noted for KA in the rec-assay and SOS spot test, respectively, chromosomal aberrations and sister chromatid exchanges were induced in Chinese hamster ovary $\mathrm{CHO}$ cells with and without $\mathrm{S} 9 \mathrm{mix}^{16}$. Chromosomal aberrations in Chinese hamster lung $\mathrm{CHL} / \mathrm{IU}$ cells were also found at $1000 \mu \mathrm{g} / \mathrm{mL} \mathrm{KA}$ in the absence of $\mathrm{S} 9 \mathrm{mix}^{26}$, but reproducibility of clastogenicity could not be confirmed at concentrations up to $5000 \mu \mathrm{g} / \mathrm{mL} \mathrm{KA}$. In addition, KA gave positive results for DNA-damaging and clastogenic ability in cultured rat liver cells ${ }^{17,18}$. Therefore, KA's contribution to genotoxicity in the induction of hepatocellular tumors in mice and rats cannot be ruled out.

In the present liver initiation assay using F344 rats, no initiation activity was observed at $2000 \mathrm{mg} / \mathrm{kg}\left(\mathrm{LD}_{50}\right.$ value $)$ of KA. Since it has been shown that genotoxic carcinogens, which do not normally target the liver, can cause induction of GST-P positive foci in an in vivo liver initiation assay ${ }^{27}$, it is generally recognized that genotoxic carcinogenesis can be detected by this initiation assay, regardless of the target organs in long-term carcinogenicity studies in rats and mice. This initiation assay seems to be a useful method for clarifying whether or not chromosome aberrations and/or gene mutations are fixed as a result of DNA damage, therefore, we examined the initiation effects of kojic acid in a short-term liver initiation assay in partially hepatectomized rats. Based on the many findings obtained by this approach, it can be concluded that KA does not have any liver initiation activity in rats, a conclusion which is supported by our present data showing that 8-OxodG formation in the liver DNA of rats exposed to KA for 28 days failed to increase. However, since it is not known whether the liver initiation assay using rats can reliably detect almost all the genotoxic carcinogens in mice, it is premature to draw a conclusion on the negativity of liver initiation activity of KA. Considering species difference between mice and rats, additional studies of the liver initiation assay using mice are now in progress to define whether KA is a genotoxic carcinogen.

In the present study, transient increases of cell proliferation in hepatocytes were observed in rats given KA for 3 and 7 days, but not for 28 days. In a previous study in which F344 rats were given diet containing 0 or $2 \% \mathrm{KA}$ for 20 weeks without any initiation treatment, liver weights 
increased without affecting the body weights in non-initiated rats receiving $2 \% \mathrm{KA}$, together with significant increases in GST-P-positive foci ${ }^{14}$. These findings may suggest that KA has a liver tumor promoting effect. A number of chemicals inducing hepatic cytochrome P-450s, particularly of the phenobarbital type, are known to be hepatic tumor promoters ${ }^{28-30}$. Although it is not known whether KA exerts phenobarbital-type induction of cytochrome P-450s, it has been demonstrated that hepatic uridine diphosphate glucuronosyl transferase was not increased in the livers of rats given $\mathrm{KA}^{31,32}$. Based on the data of that report, the tumor promoting effect of KA might not be exerted via the induction of hepatic cytochrome P-450s. Further studies are necessary to clarify the mechanism of the liver tumor promoting effect of KA.

Acknowledgements: This work was supported in part by a grant-in-aid for research on safety re-evaluation of existing food additives from the Ministry of Health, Labour and Welfare of Japan.

\section{References}

1. Parrish FW, Wiley BJ, Simmons EG, and Long L Jr. Production of aflatoxins and kojic acid by species of Aspergillus and Penicillium. Appl Microbiol. 14: 139. 1966.

2. Kwak MY and Rhee JS. Cultivation characteristics of immobilized Aspergillus oryzae for kojic acid production. Biotechnol Bioeng. 39: 903-906. 1992.

3. Saruno R, Kato F, and Ikeno T. Kojic acid, a tyrosinase inhibitor from Aspergillus albus. Agric Biol Chem. 43: 1337-1339. 1979.

4. Chen JS, Wei CI, Rolle RS, Otwell WS, Balaban MO, and Marshall MR. Inhibitory effect of kojic acid on some plant and crustacean polyphenol oxidase. J Agric Food Chem. 39: 1396-1401. 1991.

5. Kahn V. Effect of kojic acid on the oxidation of DL-DOPA, norepinephrine and dopamine by mushroom tyrosinase. Pigment Cell Res. 8: 234-240. 1995.

6. Ohyama Y. Melanogenesis-inhibitory effect of kojic acid and its action mechanism. Fragrance J. 6: 53-58. 1990.

7. Perez-Bernal A, Munoz-Perez MA, and Camacho F. Management of facial hyperpigmentation. Am J Clin Dermatol. 1: 261-268. 2000.

8. Maeda $\mathrm{K}$ and Fukuda M. In vitro effectiveness of several whitening cosmetic components in human melanocytes. J Soc Cosmet Chem. 42: 361-368. 1991.

9. Fujimoto N, Watanabe H, Nakatani T, Roy G, and Ito A. Induction of thyroid tumors in $(\mathrm{C} 57 \mathrm{BL} / 6 \mathrm{~N} \times \mathrm{C} 3 \mathrm{H} / \mathrm{N}) \mathrm{F} 1$ mice by oral administration of kojic acid. Food Chem Toxicol. 36: 697-703. 1998.

10. Takizawa T, Mitsumori K, Tamura T, Nasu M, Ueda M, Imai T, and Hirose M. Hepatocellular tumor induction in heterozygous $p 53$-deficient CBA mice by a 26-week dietary administration of kojic acid. Toxicol Sci. 73: 287-293. 2003.

11. Mitsumori K, Onodera H, Shimo T, Yasuhara K, Takagi H, Koujitani T, Hirose M, Maruyama C, and Wakana S. Rapid induction of uterine tumors with $p 53$ point mutations in heterozygous $p 53$-deficient CBA mice given a single intraperitoneal administration of $N$-ethyl- $N$-nitrosourea.
Carcinogenesis. 21: 1039-1042. 2000.

12. Onodera H, Mitsumori K, Takagi H, Yasuhara K, Koujitani T, Tamura T, and Hirose M. Susceptibility of heterozygous p53 deficient CBA mice to induction of liver proliferative lesions by phenobarbital after dimethylnitrosamine initiation. J Toxicol Pathol. 14: 273-278. 2001.

13. Takizawa T, Mitsumori K, Takagi H, Onodera H, Yasuhara K, Tamura T, and Hirose M. Modifying effects of flumequine on dimethylnitrosamine-induced hepatocarcinogenesis in heterozygous $p 53$ deficient CBA mice. J Toxicol Pathol. 14: 135-143. 2001.

14. Takizawa $T$, Imai $T$, Onose $J$, Ueda $M$, Tamura $T$, Mitsumori K, Izumi K, and Hirose M. Enhancement of hepatocarcinogenesis by kojic acid in rat two-stage models after initiation with $N$-bis(2-hydroxypropyl)nitrosamine or $N$-diethylnitrosamine. Toxicol Sci. 81: 43-49. 2004.

15. Shibuya T, Murata T, Sakamoto K, Iwahara S, and Ikeno M. Mutagenicity and dominant lethal test of kojic acid. Toxicol Sci. 7: 255-262. 1982.

16. Wei CI, Huang TS, Fernando SY, and Chung KT. Mutagenicity studies of kojic acid. Toxicol Lett. 59: 213220. 1991.

17. Kinoshita R, Ishino T, Sugiyama S, Seto T, Igarashi S, and Goetz IE. Mycotoxins in fermented food. Cancer Res. 28: 2296-2311. 1968.

18. Stark AA. Mutagenicity and carcinogenicity of mycotoxins: DNA binding as a possible mode of action. Annu Rev Microbiol. 34: 235-262. 1980.

19. Tsuda H, Lee G, and Farber E. Induction of resistant hepatocytes as a new principle for a possible short-term in vivo test for carcinogens. Cancer Res. 40: 1157-1164. 1980.

20. Sakai H, Tsukamoto T, Yamamoto M, Yanai T, Masegi T, Inada K, Nakanishi H, and Tatematsu M. Summation of initiation activities of low doses of the nonhepatocarcinogen 1,2-dimethylhydrazine in the liver after carbon tetrachloride administration. Cancer Lett. 148: 5963. 2000.

21. Dunnett CW. A multiple comparison procedure for comparing several treatments with a control. J Am Stat Association. 50: 1096-1121. 1955.

22. Gosset WS (Student). The probable error of a mean. Biometrika. 6: 1-25. 1908.

23. Dunnick JK, Hardisty JF, Herbert RA, Seely JC, FurediMachacek EM, Foley JF, Lacks GD, Stasiewicz S, and French JE. Phenolphthalein induces thymic lymphomas accompanied by loss of the $p 53$ wild type allele in heterozygous $p 53$-deficient (+/-) mice. Toxicol Pathol. 25: 533-540. 1997.

24. Tennant RW, French JE, and Spalding JW. Identifying chemical carcinogens and assessing potential risk in shortterm bioassays using transgenic mouse models. Environ Health Perspect. 103: 942-950. 1995.

25. Tennant RW, Spalding J, and French JE. Evaluation of transgenic mouse bioassays for identifying carcinogens and noncarcinogens. Mutat Res. 365: 119-127. 1996.

26. Ishidate MJr, Harnois MC, and Sofuni T. A comparative analysis of data on the clastogenicity of 951 chemical substances tested in mammalian cell cultures. Mutat Res. 195: 151-213. 1988.

27. Sakai H, Tsukamoto T, Yamamoto M, Kobayashi K, Yuasa H, Imai T, Yanai T, Masegi T, and Tatematsu M. Distinction of carcinogens from mutagens by induction of liver cell foci 
in a model for detection of initiation activity. Cancer Lett. 188: 33-38. 2002.

28. Kolaja KL, Stevenson DE, Walborg EFJr, and Klaunig JE. Dose dependence of phenobarbital promotion of preneoplastic hepatic lesions in $\mathrm{F} 344$ rats and $\mathrm{B} 6 \mathrm{C} 3 \mathrm{~F} 1$ mice: effects on DNA synthesis and apoptosis. Carcinogenesis. 17: 947-954. 1996.

29. Shoda T, Mitsumori K, Onodera H, Toyoda K, Uneyama C, Takada K, and Hirose M. Liver tumor-promoting effect of beta-naphthoflavone, a strong CYP 1A1/2 inducer, and the relationship between CYP $1 \mathrm{~A} 1 / 2$ induction and $\mathrm{C} \times 32$ decrease in its hepatocarcinogenesis in the rat. Toxicol Pathol. 28: 540-547. 2000

30. Kinoshita A, Wanibuchi H, Imaoka S, Ogawa M, Masuda C, Morimura K, Funae Y, and Fukushima S. Formation of 8- hydroxydeoxyguanosine and cell-cycle arrest in the rat liver via generation of oxidative stress by phenobarbital: association with expression profiles of $\mathrm{p} 21^{\mathrm{WAF} 1 / \mathrm{Cip} 1}$, cyclin D1 and Ogg1. Carcinogenesis. 23: 341-349. 2002.

31. Mitsumori $\mathrm{K}$, Onodera $\mathrm{H}$, Takahashi $\mathrm{M}$, Funakoshi $\mathrm{T}$, Tamura T, Yasuhara K, Takegawa K, and Takahashi M. Promoting effects of kojic acid due to serum TSH elevation resulting from reduced serum thyroid hormone levels on development of thyroid proliferative lesions in rats initiated with $\mathrm{N}$-bis(2-hydroxypropyl)nitrosamine. Carcinogenesis. 20: 173-176. 1999.

32. Tamura T, Mitsumori K, Onodera H, Fujimoto N, Yasuhara $\mathrm{K}$, Takegawa $\mathrm{K}$, and Takahashi $\mathrm{M}$. Inhibition of thyroid iodine uptake and organification in rats treated with kojic acid. Toxicol Sci. 47: 170-175. 1999. 